\title{
First come, first served: superinfection exclusion in Deformed wing virus is dependent upon sequence identity and not the order of virus acquisition
}

\author{
Olesya N. Gusachenko (D) ${ }^{1 凶}$, Luke Woodford (iD) ${ }^{1}$, Katharin Balbirnie-Cumming ${ }^{1}$ and David J. Evans (iD) \\ (c) The Author(s) 2021
}

\begin{abstract}
Deformed wing virus (DWV) is the most important globally distributed pathogen of honey bees and, when vectored by the ectoparasite Varroa destructor, is associated with high levels of colony losses. Divergent DWV types may differ in their pathogenicity and are reported to exhibit superinfection exclusion upon sequential infections, an inevitability in a Varroa-infested colony. We used a reverse genetic approach to investigate competition and interactions between genetically distinct or related virus strains, analysing viral load over time, tissue distribution with reporter gene-expressing viruses and recombination between virus variants. Transient competition occurred irrespective of the order of virus acquisition, indicating no directionality or dominance. Over longer periods, the ability to compete with a pre-existing infection correlated with the genetic divergence of the inoculae. Genetic recombination was observed throughout the DWV genome with recombinants accounting for $\sim 2 \%$ of the population as determined by deep sequencing. We propose that superinfection exclusion, if it occurs at all, is a consequence of a cross-reactive RNAi response to the viruses involved, explaining the lack of dominance of one virus type over another. A better understanding of the consequences of dual- and superinfection will inform development of cross-protective honey bee vaccines and landscape-scale DWV transmission and evolution.
\end{abstract}

The ISME Journal (2021) 15:3704-3713; https://doi.org/10.1038/s41396-021-01043-4

\section{INTRODUCTION}

Honey bees (Apis mellifera) are globally important pollinators of wild flowers and agricultural crops, and the source of honey, with annual global production worth in excess of $\$ 7 \mathrm{bn}[1]$. Both honey production and pollination services require strong, healthy colonies, which are threatened by a range of factors, but most significantly by disease. One of the major viral pathogens of honey bees is Deformed wing virus (DWV). When transmitted by the parasitic mite Varroa destructor, DWV is responsible for high overwinter colony losses, which can exceed $37 \%$ annually [2]. Improvements to honey bee health, through direct control of virus transmission or replication, require a better understanding of how the virus propagates within and between bees.

The historical identification and naming of DWV-like viruses imply a greater genetic divergence than subsequent molecular analysis has demonstrated. In 2004-2006 several picorna-like viruses with high levels of sequence identity were reported [3-5]. These viruses were initially named according to their origins; the virus from honey bees with characteristic wing deformities was termed DWV [4], a similar virus found in aggressive workers in Japan was designated Kakugo virus [3,5] and analysis of Varroa mites yielded Varroa destructor virus type 1 (VDV-1) [3]. Limited genetic divergence ( 84-97\% genomic RNA identity), similar infectivity in honey bees, and demonstrated ability to freely recombine during coinfections [6-9] resulted in them now being considered as different variants of DWV $[6,7,10]$, albeit occupying two genetic branches (VDV-1-like and DWV-like) of the same phylogenetic tree [11]. To distinguish between these branches the terminology 'type $A$ ' and 'type $B$ ' has been adopted for DWV-like and VDV-1-like variants respectively. Evidence for the existence of a third type named DWV type $C$ has also been reported [12].

DWV is ubiquitous in honey bees [13-15], with the possible exception of Australian colonies [16]. In the absence of Varroa the virus is transmitted horizontally, per os, and vertically from the infected queen and the drones [17]. With subsequent Varroa mite transmission it is therefore inevitable that the virus enters a host already harbouring one or multiple DWV variants. Current studies suggest that DWV infection can occur with several variants cocirculating in the same apiary, colony, or individual honey bee host [18-21]. Although the type A and B variants appear to be differentially distributed, with type A frequently reported in the US and type $\mathrm{B}$ being commonly detected in European colonies $[8,13,22]$, direct competition may occur where they cocirculate. If this competition has a directionality, it will influence the distribution and future spread of DWV at the landscape scale. While some studies of mixed DWV infections demonstrate no predominance of one variant over another [18, 23], others show possible competition between the variants and higher accumulation of DWV B in infected bees [24]. In addition, superinfection exclusion (SIE) has been proposed, in which a pre-existing type $B$ virus prevents the establishment of a type $A$ infection at the colony level [10].

${ }^{1}$ Biomedical Sciences Research Complex, University of St. Andrews, North Haugh, St. Andrews, UK. ${ }^{\times}$email: olesya.gusachenko@gmail.com 
A recently developed reverse genetics (RG) system comprising a set of genetically tagged DWV variants and reporter geneexpressing viruses provides an opportunity to investigate coinfection kinetics and competition between DWV types [25]. Since SIE is a widely observed virological phenomenon [26-35], we extended these studies to assay dominance of one variant over another during sequential infection. Using reporter gene-expressing DWV we additionally investigated the influence of competition on tissue distribution of infection. We show that where competition is observed, manifested as reduced virus levels, it is reflected in reduced reporter gene expression at the cellular level. Notably we show that DWV accumulation during superinfection is influenced by the genetic identity between the viruses, rather than by a directionality of competition. Genetically divergent DWV variants (such as those representing type A and type B) exhibit transient competition, whilst viruses with greater identity (e.g. type $A / B$ recombinants with either type $A$ or type $B$ ) demonstrate a distinctly more pronounced effect. We also analysed the occurrence and identity of recombinants during mixed infections and confirmed that these are present with junctions widely distributed throughout the genome. These studies provide further insights into the biology of DWV. In particular they address the consequences of coand superinfection, an important consideration when transmitted by the ectoparasite Varroa. Our results indicate that genome identity is the determinant that defines the outcome of dual infections; this will inform studies of population transmission at the landscape scale and possible future developments of 'vaccines' to protect honey bees from viral disease [36].

\section{MATERIALS AND METHODS}

\section{RG DWV clones preparation}

VDD, VVD and VVV RG constructs used in this study were described earlier [25], DDD RG cDNA was prepared by modification of the VDD RG system with a DWV type A parental sequence insert, which was based on published data [37] and obtained by custom gene synthesis (IDT, Leuven, Belgium). Enhanced green fluorescent protein (EGFP) and mCherryexpressing chimeric DWV genomes were built via incorporation of the reporter-encoding sequence into DWV cDNA as described previously [25]. All plasmid sequences were verified by Sanger sequencing. CDNA sequences of DDD, $\mathrm{WVV}_{\mathrm{mc}}$, and $\mathrm{WVD}_{\mathrm{s}}$ are shown in Text S1, other RG cDNAs are available online (GenBank accession numbers: DWV-VDD MT415949, DWV-VVD (VVD $)$ - MT415950, DWV-VVV - MT415952, DWVVDD-eGFP - MT415948, DWV-VVD-eGFP - MT415953).

\section{Viral RNA and siRNA synthesis}

DWV RNA was synthesised from linearised plasmid templates with T7 RiboMAX Express Large Scale RNA Production System (Promega, Southampton, UK), and purified with the GeneJet RNA Purification Kit (Thermo Fisher Scientific) as described in [25].

siRNA strands were prepared using Express Large Scale RNA Production System (Promega) according to the manufacturer's protocol with double stranded DNA templates annealed from synthetic oligonucleotide pairs containing T7 RNA polymerase promoter sequence (Table S1).

\section{Viruses}

Infectious DWV was prepared from honey bee pupae injected with in vitro generated RNA as previously described $[25,38]$. For quantification RNA was extracted from $100 \mu \mathrm{l}$ of virus preparation using the RNeasy kit (Qiagen, Manchester, UK) and analysed by reverse transcription and quantitative PCR (qPCR).

\section{Honey bees and bumble bees}

All honey bee (Apis mellifera) brood in this study was obtained from the University of St Andrews research apiary. Colonies were managed to reduce Varroa levels and endogenous DWV levels were regularly tested. Honey bee larvae and both honey and bumble bee pupae (Bombus terrestris audax, Biobest, Belgium) were maintained and fed as described previously $[25,38]$.

\section{Virus inoculations}

Virus injections of pupae were performed with insulin syringes (BD Micro Fine Plus, $1 \mathrm{ml}, 30 \mathrm{G}$, Becton Dickinson, Oxford, UK) as described in [25, 38]. Oral larval infection was carried out by single DWV feeding according to the previously described procedure [25].

\section{RNA extraction, reverse transcription and PCR (RT-PCR)}

RT-PCR and qPCR analysis of individual pupae samples was performed as previously described [25]. Sequences of primers are shown in Table S1. When required, PCR products were subjected to restriction digest prior to loading on the $1 \%$ agarose gels stained with ethidium bromide. DWV titres were calculated by relating the resulting $\mathrm{Ct}$ value to the standard curve generated from a serial dilution of the CDNA obtained from the viral RNA used for virus stock preparation.

\section{Microscopy}

Imaging was conducted using a Leica TCS SP8 confocal microscope with $10 \times$ HC PL FLUOTAR objective. For dissected pupae analysis samples were mounted in a drop of PBS under the microscope cover slides and observed by microscopy within $1 \mathrm{~h}$ after the dissection.

\section{Sample libraries for next generation sequencing}

RNA was reverse transcribed using Superscript III polymerase (Invitrogen, Thermo Fisher Scientific) with DWV FG RP1 primer (Table S1) using $1 \mu \mathrm{g}$ of total RNA in a $20 \mu \mathrm{l}$ final reaction volume and following the manufacturer's protocol. Reactions were incubated at $50^{\circ} \mathrm{C}$ for $1 \mathrm{~h}, 75^{\circ} \mathrm{C}$ for 15 min.

The transcribed cDNA was amplified using LongAmp Taq polymerase (New England Biolabs) to produce $a \sim 10 \mathrm{~Kb}$ PCR fragment. The reactions were carried out according to the manufacturer's protocol with the following thermal profile: $30 \mathrm{~s}$ at $95^{\circ} \mathrm{C}, 30$ cycles of $95^{\circ} \mathrm{C}$ for $15 \mathrm{~s}, 53^{\circ} \mathrm{C}$ for $30 \mathrm{~s}$, and $65^{\circ} \mathrm{C}$ for $8 \mathrm{~min}$, with a final extension at $65^{\circ} \mathrm{C}$ for $10 \mathrm{~min}$.

\section{Recombination analysis}

Purified amplicons were sequenced using an HiSeq (Illumina, Cabmridge, UK) at the University of St Andrews, producing $2 \times 300 \mathrm{bp}$ paired-end reads. The sequences were converted to FASTA format, extracted and trimmed using Geneious (v.2019.1.3). A reference genome file was made using VVV and VDD CDNA sequences with a terminal pad of A-tails added to maximise sensitivity [39]. The reference file was indexed using Bowtie Build (Version 0.12.9) and the reads were mapped to the reference file using the recombinant-mapping algorithm, ViReMa (Viral-Recombination Mapper, Version 0.15). The recombinant sequences were compiled as a text file and analysed using ggpubr (v2.3) in R Studio.

\section{RESULTS}

\section{Modular RG system design for DWV}

To compare the virulence and competitiveness of DWV types and their recombinants a set of cDNA clones were prepared. By exploiting the modular organisation of the DWV genome $[9,21]$ we have previously constructed infectious cDNAs for several distinct genetic variants of DWV [25]. For convenience these are referred to as follows: VDD (DWV type A coding sequence, GenBank MT415949), VVD (a type B/A recombinant, GenBank MT415950), and VVV (DWV type B, GenBank MT415952). In addition, we constructed a cDNA for a complete type A DWV, designated DDD, using a similar gene synthesis and module replacement strategy [25] to incorporate the DWV type A 5'untranslated region (5'-UTR; DWV-A 1414, GenBank KU847397 used as a reference-Fig. S1). VDD, VVV, and VVD DWV variants were previously shown to be infectious and cause symptomatic disease in honey bees [25]. Infectivity of the DDD virus was verified by analysis of DWV accumulation in injected pupae and was indistinguishable from the VDD virus (Fig. S2a). Derivatives of VDD, VVD, and VVV, expressing EGFP or mCherry, were generated as previously described [25] (Fig. S1) and their replication verified following inoculation of pupae (for example, Fig. S2b). 
a.

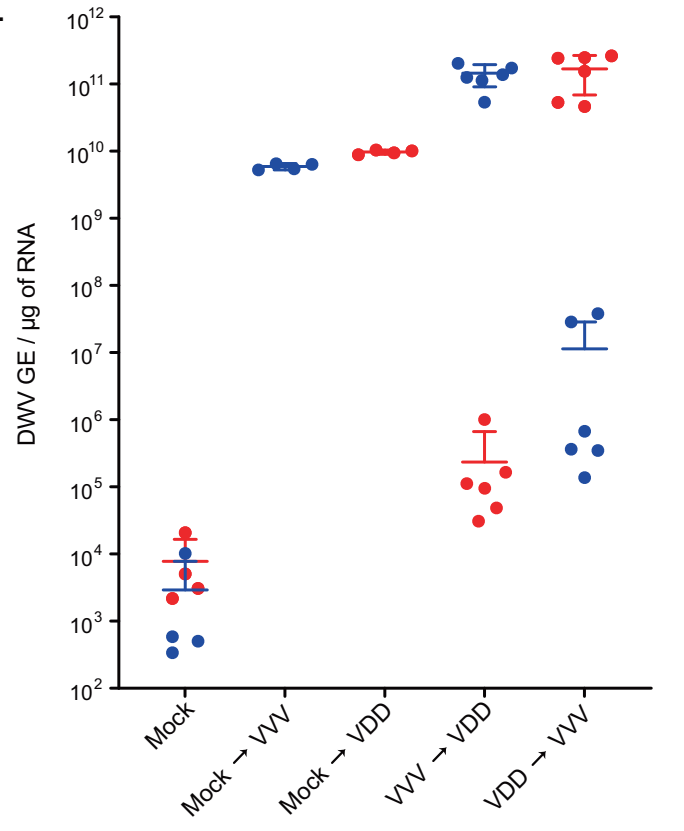

b.

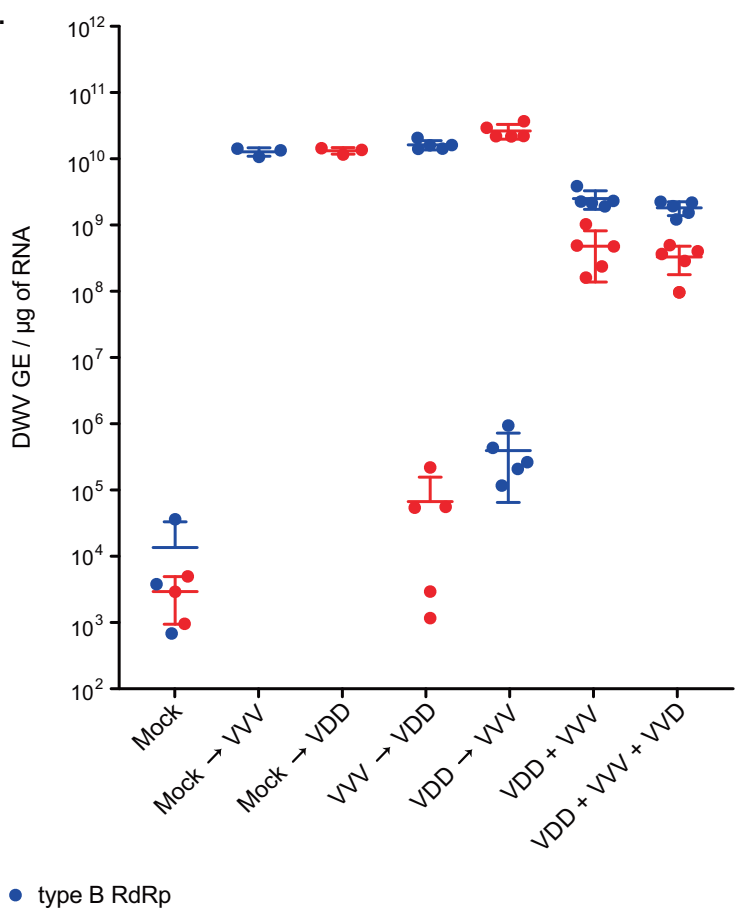

Fig. 1 Coinfection and superinfection of honey bee brood with DWV. a Superinfection of honey bee pupae preliminarily infected per os at larval stage and then via injection at pupal stage. Quantified viral titres of VVV and VDD DWV in pupae $24 \mathrm{~h}$ post-injection of the superinfecting virus are shown (second virus inoculated by injection after primary infection by feeding with the reciprocal DWV variant is indicated by " $\rightarrow$ ", e.g. VVV (fed) $\rightarrow$ VDD (injected)). b DWV accumulation in honey bee pupae in coinfected (mixed virus population indicated by " + ") or superinfected samples (second injection $24 \mathrm{~h}$ after primary infection by injection is indicated by " $\rightarrow$ "). Primer pairs for type A or type B RdRp amplifying variant-specific fragments of virus polymerase encoding region were used to distinguish between the administered variants. Data points represent DWV levels in individual pupae with two points of different colour corresponding to different virus variants (red for type A RdRp and blue for type B RdRp respectively) in the same pupa (or in individual pupae for VVV or VDD only injected samples). Error bars show mean \pm SD for each virus variant in each injection group, GE-genome equivalents. ANOVA: $p<0.05$ for type A accumulation in "Mock $\rightarrow$ VDD" vs. "VVV $\rightarrow$ VDD", for type B in "Mock $\rightarrow$ VV" vs. "VDD $\rightarrow$ VV" groups, for type A vs. type B in "VVV + VDD" and "VVV + VVD + VDD" groups.

\section{Superinfection and coinfection studies}

Varroa delivers DWV to developing honey bee pupae by direct injection when feeding. Pupae will already contain previously acquired DWV and the mite may contain one or more DWV variants. We investigated the consequences of coinfection and superinfection on accumulation of distinct DWV variants in honey bee pupae under laboratory conditions. Primary infection was achieved by feeding first instar larvae ( $0-1$ day old) with a diet containing $10^{7}$ genome equivalents (GE) of either VDD or VVV DWV, followed by secondary inoculation by injection $\left(10^{3} \mathrm{GE}\right)$ with the reciprocal virus variant 10 days later at the white-eyed pupal stage. The viral load in individual pupae was analysed by qPCR $24 \mathrm{~h}$ post-injection using DWV type-specific primers for the RNAdependent RNA polymerase (RdRp) coding region. In the absence of superinfection, larvae fed either VDD or VVV showed accumulation of the virus to levels of $\sim 10^{10} \mathrm{GE} / \mu \mathrm{g}$ RNA by the time of pupation (Fig. S2c). Pupae initially infected by larval feeding and subsequently superinfected by injection showed a markedly reduced accumulation of the injected DWV variant when compared to the same virus in pupae which were not fed DWV as larvae (Fig. 1a).

Reduced accumulation of a superinfecting virus was also observed when white-eyed pupae were initially injected with VDD or VVV $24 \mathrm{~h}$ prior to introduction of the reciprocal virus variant (first injection- $10^{2} \mathrm{GE}$, superinfection $-10^{6} \mathrm{GE}$, Fig. 1b). In contrast, simultaneous infection with two or three (VDD, VVV and VVD) DWV variants $\left(10^{2} \mathrm{GE}\right.$ in total virus injected corresponding to $0.5 \times 10^{2}$ or $0.33 \times 10^{2} \mathrm{GE}$ of each variant for two- and threecomponent infections respectively) resulted in nearly equivalent virus loads, although the VDD variant accumulated to slightly lower $\left(\sim 0.5 \log _{10}\right)$ titres at $24 \mathrm{~h}$ post-injection.

\section{Dynamics of DWV accumulation in superinfection conditions} We extended these studies to determine whether the apparent competitive disadvantage for the second virus remained after an extended incubation period. Pupal injections were repeated as before and viral loads quantified 5 and 7 days after superinfection. A recombinant type $B / A$ variant (VVD) was additionally included both as primary and superinfecting virus. In reciprocal infections using VDD and VVV both the initial and the superinfecting virus reached nearly equivalent levels within the incubation period (Fig. 2). In contrast, in virus pairings with a greater sequence identity between the genomes the superinfecting virus exhibited a reduced accumulation even after prolonged incubation. In the "VDD $\rightarrow$ VVD", "VVD $\rightarrow$ VDD", and "VVD $\rightarrow$ VVV" groups the superinfecting virus levels were $\sim 2 \log _{10}$ lower than the initial inoculum at 5-7 days post-injection. For the "VVV $\rightarrow$ VV" pairing this was more marked, with the superinfecting virus still $\sim 4 \log _{10}$ lower after 7 days. In control pupae infected with VDD, VVD or VVV individually all three viruses reached high titres 7 days postinjection (Fig. 2 and Fig. S3). In addition, virus accumulation was monitored after coinfection of equal amounts of each combination of VDD, VVD and VV over time. In these studies, all coinfecting variants achieved similar titres 5 days post-inoculation (Fig. S3).

We recently demonstrated that bumble bees are susceptible to DWV infection when directly injected at high doses [38]. We therefore investigated the influence of the host environment on 


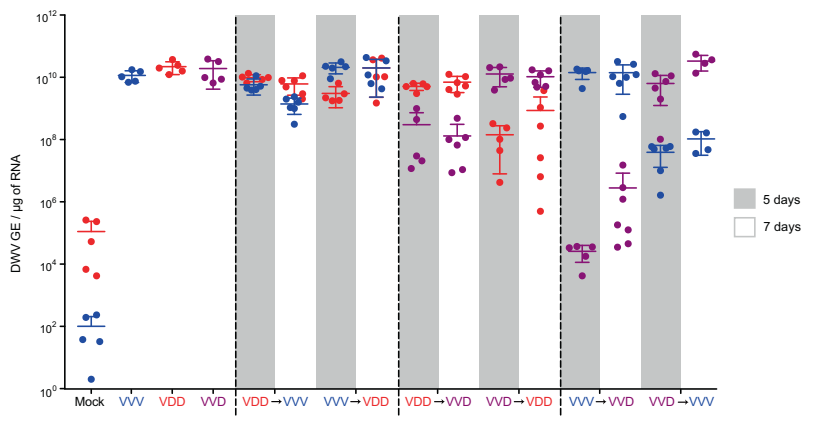

Fig. 2 qPCR analysis of DWV accumulation in superinfection conditions. Honey bee pupae received a primary injection with one DWV variant (VVV, VDD or VVD) and a secondary injection (superinfection) with a different variant $24 \mathrm{~h}$ later. DWV accumulation was quantified 5 (grey shading) and 7 (no shading) days after the second injection. Primer sets specifically targeting the viral polymerase or a structural protein encoding region of DWV type $A$ or type B were used to detect accumulation of each of the injected variants. Data points represent DWV levels in individual samples with two points of different colour corresponding to different virus variants (red for VDD, blue for VVV, and purple for VVD respectively) in the same pupa (or in individual pupae for VVV, VDD or VVD only injected samples). Error bars show mean \pm SD for each virus variant in each injection group, GE-genome equivalents. ANOVA: $p<0.05$ for "Mock $\rightarrow$ VDD" vs. "VVD $\rightarrow$ VDD", "Mock $\rightarrow$ VVD" vs. "VDD $\rightarrow$ VVD", "Mock $\rightarrow$ VVD" vs. "VVV $\rightarrow$ VVD", "Mock $\rightarrow$ VVV" vs. "VVD $\rightarrow$ VV" at 7 days time point.

the DWV superinfection by conducting similar experiments in bumble bee pupae. At $48 \mathrm{~h}$ post superinfection the levels of the second virus administered were lower than that of the primary virus inoculated but-with the exception of the "VVD $\rightarrow \mathrm{VVV}^{\prime}$ combination-had achieved similar levels by 6 days post-injection (Fig. S4).

These results suggest that a superinfecting virus experiences an initial competitive disadvantage, but that this disadvantage is overcome after 5-7 days unless the viruses exhibit more extensive sequence identity. To investigate this further we studied superinfection with essentially identical viruses, using two VVD variants distinguishable solely by unique genetic tags $-\mathrm{VVD}_{\mathrm{S}}$ and $\mathrm{VVD}_{\mathrm{H}}$, tagged with a Sall or Hpal restriction site respectively (Fig. S1, Text S1)—which differ by just four nucleotides. Honey bee pupae injected with $\mathrm{VVD}_{\mathrm{H}}$ were challenged $24 \mathrm{~h}$ later with $\mathrm{VVD}_{\mathrm{S}}$ and analysed by end point PCR and restriction assay 1, 3 and 6 days after superinfection. No $V_{V D}$ was detectable in superinfected pupae at any time point analysed (Fig. S5) suggesting a complete or near-complete block of the superinfecting genome amplification. Control injections of $\mathrm{VVD}_{\mathrm{S}}$ into pupae, which did not receive $\mathrm{VVD}_{\mathrm{H}}$ virus, allowed detection of Sall-tagged CDNA $24 \mathrm{~h}$ postinoculation.

\section{Tissue localisation studies using reporter-encoding DWV}

Total RNA levels analysis allows the quantification of DWV to be determined, but it obscures details of the relative distribution and tissue tropism of individual virus variants. Previously we developed an EGFP-encoding RG system for DWV [25] based upon the VDD genome and designated $\operatorname{DWV}_{\mathrm{E}}$ (for convenience here renamed to $\left.V D D_{E}\right)$. We used $V D D_{E}$ to define whether the primary infection also affects the distribution of the superinfecting virus. Furthermore, we constructed a full length DWV type A genome, designated DDD (Fig. S1), and similarly investigated superinfection of DDD infected pupae. Pupae that had received an initial injection of $10^{2} \mathrm{GE}$ of DDD, VDD, VVD or VVV were inoculated $24 \mathrm{~h}$ later with $10^{6} \mathrm{GE}$ of $\mathrm{VDD}_{\mathrm{E}}$. Live pupae were analysed by confocal microscopy for the presence of the EGFP signal (Fig. 3). Three $r-$ egions of each pupa were visualised-the head, the developing wing and the abdomen-as we have previously demonstrated significant virus accumulation in these locations [25].

Injection of $\mathrm{VDD}_{\mathrm{E}}$ in the absence of a primary infection ("Mock $\rightarrow V^{\prime} D_{E}$ " group) resulted in efficient expression of EGFP throughout the pupa $24 \mathrm{~h}$ post-inoculation (Fig. $3 \mathrm{a}-\mathrm{c}$ ). In the case of superinfection, the EGFP signal could be seen $24 \mathrm{~h}$ later only in pupae where VVV was used as a primary infecting genotype (Fig. $3 \mathrm{~h}$ and i). In these pupae, the number of fluorescent foci was lower when compared to the "Mock $\rightarrow V D_{E}$ " group infected for the same $24 \mathrm{~h}$ period (Fig. 3, panels a-c vs. $\mathrm{g}$-i). No EGFP signal was visible upon superinfection with $V_{D D}$ after $24 \mathrm{~h}$ in pupae first injected with VVD, VDD and DDD (data not shown). At 4-5 days post-inoculation with $\mathrm{VDD}_{\mathrm{E}}$ there were also differences observed in the levels and distribution of the reporter protein. For example, no EGFP signal was found in the wings after primary inoculation with VVD or DDD (Fig. 3, panels $\mathrm{n}$ and $\mathrm{t}$ vs. e and k). Visible EGFP expression was detected in the head and abdomen in the pupae from these injection groups after 4-5 days but the extent and number of fluorescent foci was reduced when compared to the "Mock $\rightarrow \mathrm{VDD}_{\mathrm{E}}$ " and "VVV $\rightarrow \mathrm{VDD}_{\mathrm{E}}$ " pupae (Fig. 3, panels $\mathrm{m}, \mathrm{o}, \mathrm{s}$ and $u$ vs. panels $d, f, j$ and I). In contrast to the "Mock $\rightarrow V D D_{E}$ " and "VVV $\rightarrow V D_{E}$ " samples, only a fraction of pupae in "VVD $\rightarrow V D_{E}$ " and "DDD $\rightarrow V D_{E}$ " groups exhibited detectable EGFP signal in each of the body sites under analysis (Table S2). Finally, pupae initially injected with VDD did not show any detectable EGFP signal even 6 days after superinfection with $\operatorname{VDD}_{E}$ (Fig. 3p-r, Fig. S6), suggesting again that greater sequence identity restricts the activity of the superinfecting virus.

To confirm that the external analysis of the intact living pupae was representative, selected samples were dissected. Tissue samples, including parts of the digestive tract, wing rudiments, thoracal muscle tissue, brain and cephalic glands were visualised by confocal microscope (Fig. S6). This analysis recapitulated the pattern of fluorescence observed by previous visualisation of intact pupae. To complement the microscopy data, we quantified DWV RNA in selected pupae by qPCR at $24 \mathrm{~h}$ and 5 days post superinfection (Fig. S7). Analysis by $\mathrm{QPCR}$ revealed that a proportion of the $V_{D D}$ population has lost the non-essential EGFP-coding sequences evidenced by the EGFP/RdRp ratio in the "Mock $\rightarrow V D_{E}$ " group. This instability of EGFP-expressing viruses has previously been reported for DWV [40] and poliovirus [41]. However, sufficient EGFP-expressing reporter viruses remained in the population to allow their detection by microscopy and RNA quantification 5 days post-injection. The results of qPCR have shown that there was a good agreement between the amount of genomic RNA and the observed level of fluorescence.

\section{Localisation of DWV in coinfected and superinfected pupae using two-colour microscopy}

In order to visualise the distribution of infection with different DWV variants we used EGFP- and mCherry-expressing viruses, $\mathrm{VDD}_{\mathrm{E}}, \mathrm{VVD}_{\mathrm{E}}$, and $\mathrm{VVV}_{\mathrm{mc}}$ (with subscript $\mathrm{E}$ and $\mathrm{mC}$ indicating the EGFP or mCherry reporter respectively, Fig. S1). For coinfection, pupae were injected with equimolar mixtures of $V V D_{E}$ or $V D D_{E}$ and $\mathrm{WVV}_{\mathrm{mc}}$ and analysed under the confocal microscope 1-5 days post-inoculation. We could readily detect red and green fluorescent signals present in the same tissues of virus-injected pupae as previously described [25], including multiple tissues of the digestive tract, wings and head tissues. The reporter gene expression sites appeared as individual punctate foci of either red or green fluorescence, with only a few displaying dual fluorescence for both reporters (Fig. $4 \mathrm{a}$ and Fig. S8). The analysis of $\mathrm{VDD}_{\mathrm{E}}$-infected pupae superinfected with $\mathrm{VVV}_{\mathrm{mc}}$ and visualised by microscopy after a further $24 \mathrm{~h}$ revealed a similar distribution of the fluorescent signal as in coinfected samples (Fig. 4b). 

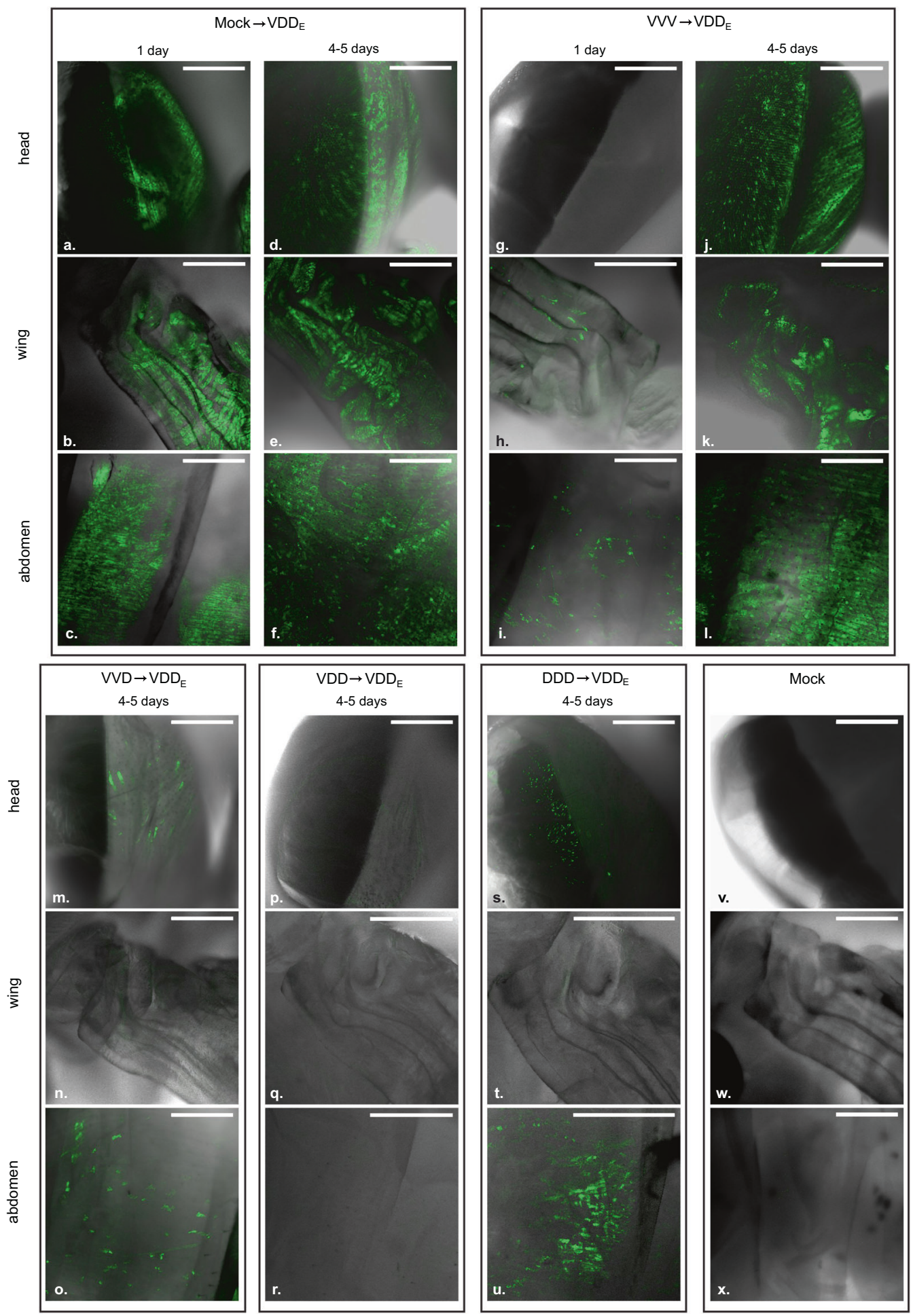

Fig. 3 EGFP signal localisation in $\mathrm{VDD}_{\mathrm{E}}$ injected honey bee pupae analysed by confocal microscopy. Combined white-field and fluorescent images are shown for convenience of interpretation. Pupae were analysed 1 and 4-5 days after the second injection. For VVD, VDD and DDD primary infection groups only samples incubated for 4-5 days are shown, as no EGFP was detected after 1 day. Scale bars correspond to $500 \mu$ m.

\section{Recombination between VDD and VVV DWV}

The interpretation of the superinfection studies is based upon sequence-specific quantification of particular regions of the virus genome by qPCR. This interpretation could be confounded by extensive levels of genetic recombination, a natural consequence of coinfection with related viruses [42]. Genetic recombination of RNA viruses requires that both parental genomes are present within an individual cell [43]. Since our microscopy analysis had detected only limited numbers of apparently dually infected foci during mixed infections, we conducted further analysis to investigate the presence and identity of viral recombinants, and the influence of the order of virus acquisition on recombination, using next generation sequencing. Illumina paired-reads were generated from PCR amplicons of a $10 \mathrm{~Kb}$ fragment of the DWV genome targeting pupae initially 
a.

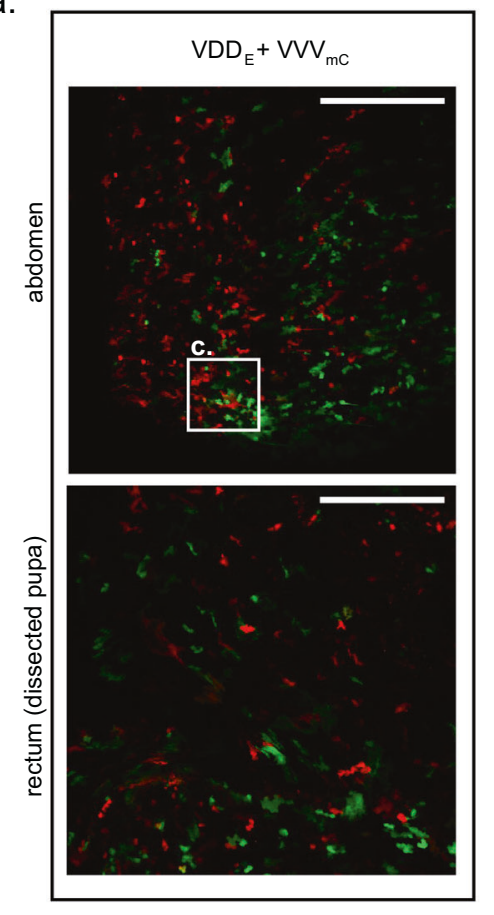

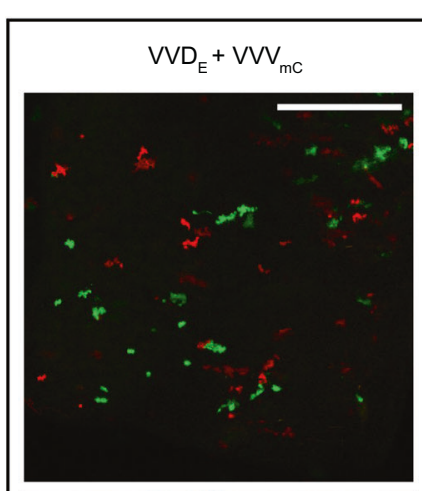

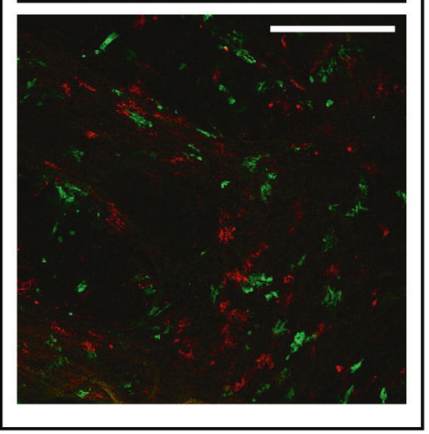

b.

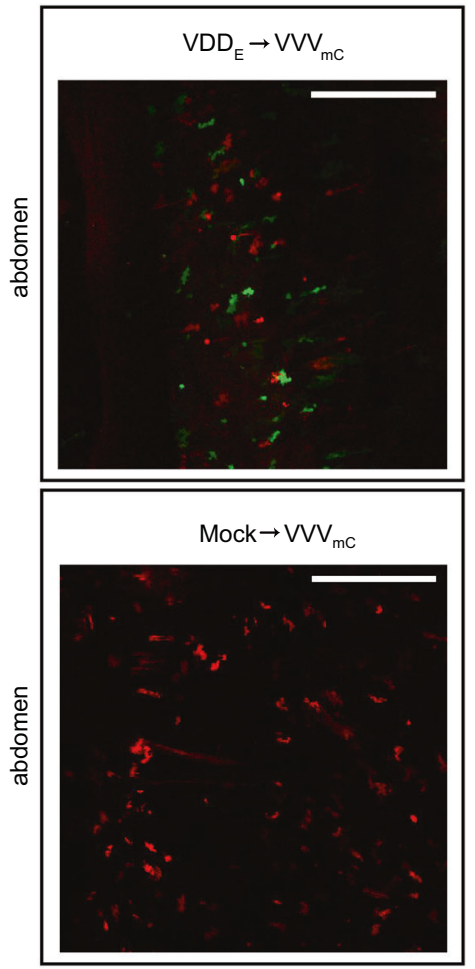

C.

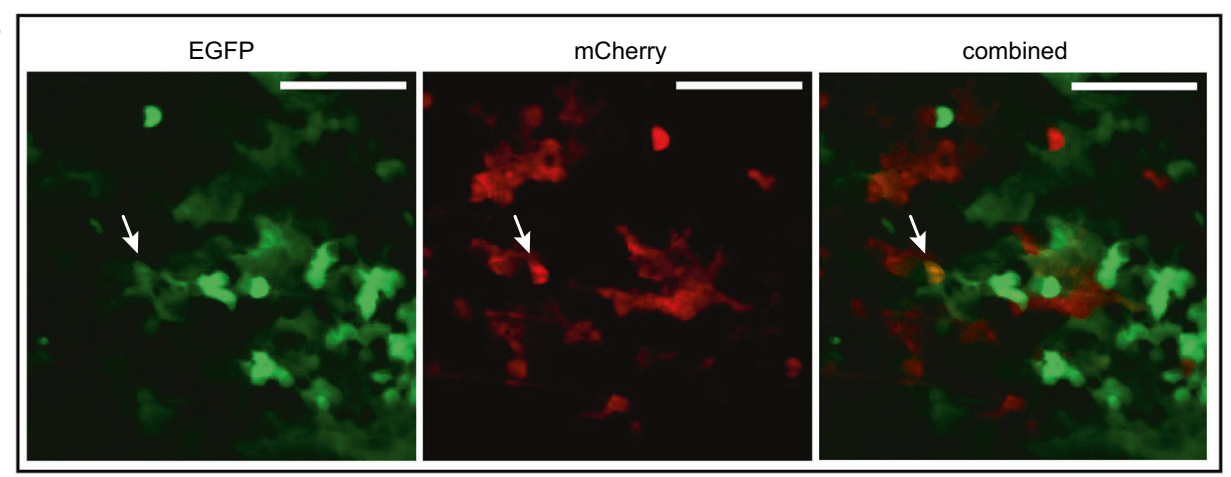

Fig. 4 Confocal microscopy analysis of honey bee pupae coinfected or superinfected with DWV variants encoding EGFP and mCherry. a Coinfection: " $\mathrm{VDD}_{\mathrm{E}}+\mathrm{VVV}_{\mathrm{mc}}$ " or " $\mathrm{VVD}_{\mathrm{E}}+\mathrm{VVV}_{\mathrm{mc}}$ " panels show red (mCherry) and green (EGFP) fluorescence signals detected in the abdomen of intact pupae (upper row) or in the dissected tissues of the digestive tract (rectum tissue shown as an example). Scale bars correspond to $500 \mu \mathrm{m}$. b Superinfection: upper panel-abdomen of an intact pupa initially infected with $\mathrm{VDD}_{\mathrm{E}}$ and superinfected $24 \mathrm{~h}$ later with VV $\mathrm{V}_{\mathrm{mc}}$ analysed $24 \mathrm{~h}$ after the second injection; lower panel-abdomen of an intact pupa injected with $\mathrm{VVV}_{\mathrm{mc}}$ only and analysed $24 \mathrm{~h}$ postinoculation. Scale bars correspond to $500 \mu \mathrm{m}$ c Magnified image of highlighted region (from a) of pupa coinfected with $\mathrm{VDD}_{\mathrm{E}}+\mathrm{VVV}_{\mathrm{mc}}$. Individual images for EGFP and mCherry signals, and a combined image for both fluorophores are shown. Arrows indicate individual foci of infection exhibiting both EGFP and mCherry expression. Scale bars correspond to $100 \mu \mathrm{m}$.

infected with VDD and challenged with WV, or vice versa (samples "VW $\rightarrow$ VDD" and "VDD $\rightarrow$ VV" at 5 or 7 days after superinfection). Recombination junctions were detected across the entirety of the DWV genome in all samples analysed with 'hotspots' of recombination denoted by an increased number of aligned reads identified at numerous points in the genome (Fig. 5b, Table S3), including some previously reported [21]. The percentage of reads corresponding to recombination junctions varied in individual pupae from $1-2.2 \%$ of all mapped reads (Fig. S9). In all cases approximately equal proportions of recombinants were detected with VW or VDD as the $5^{\prime}$-acceptor partners (terminology assumes that recombination occurs during negative strand synthesis $[42,44])$. In several instances we detected the same recombination junction with both $\mathrm{WV}$ and VDD as the $5^{\prime}$-acceptor. Our analysis also revealed recombination sites in which the $5^{\prime}$ was only ever derived from one variant or the other (red and blue points in Fig. 5b, Table S3). These results demonstrate that although superinfecting virus recombines readily with an established variant, the recombinant population remains a minor component of the total virus population, and is well below the level expected to confound our analysis of competition between extant and superinfecting viruses.

\section{DISCUSSION}

The global distribution and ubiquitous nature of DWV [14, 19], transmitted vertically and horizontally in honey bees $[15,45,46]$, inevitably means that when vectored by Varroa it is introduced to the host as a superinfecting virus. As such, there is the potential for competition for cellular resources in coinfected tissues, or the possibility of a pre-existing infection retarding or inhibiting superinfection through molecular mechanisms including SIE or the immune responses induced by the initial 
a.

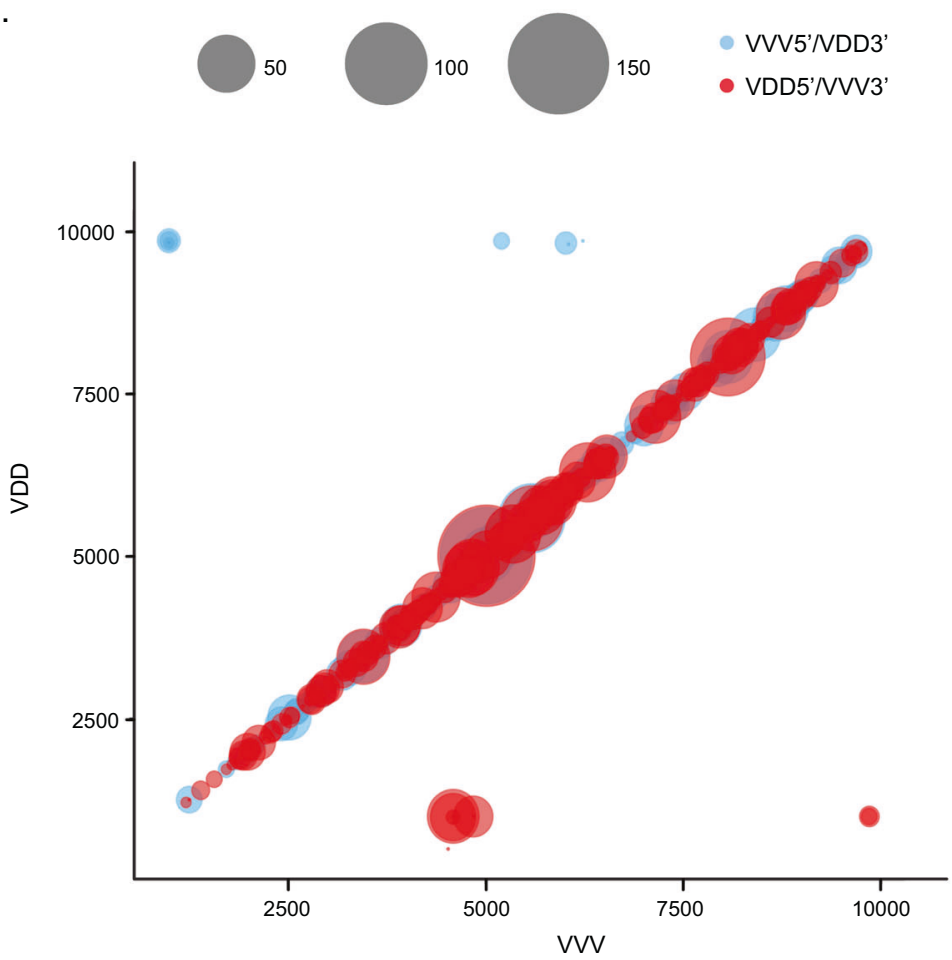

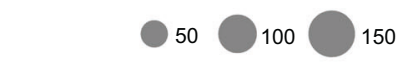
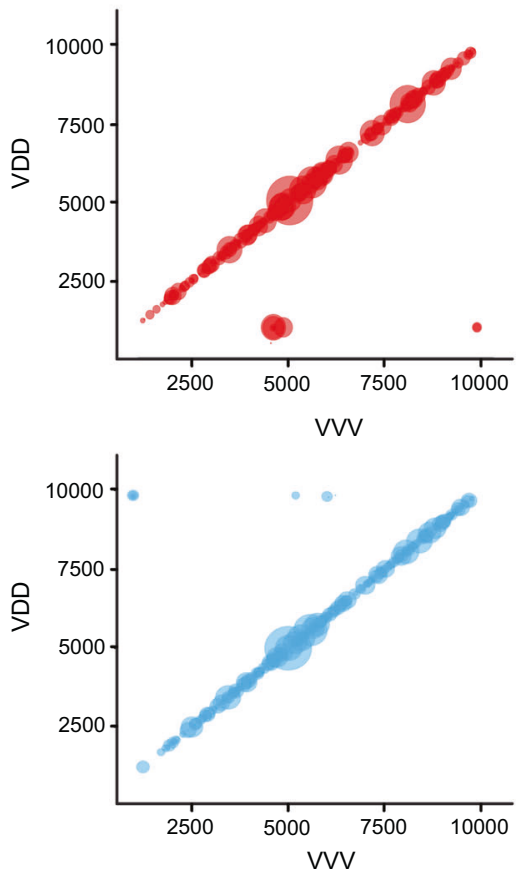

b.

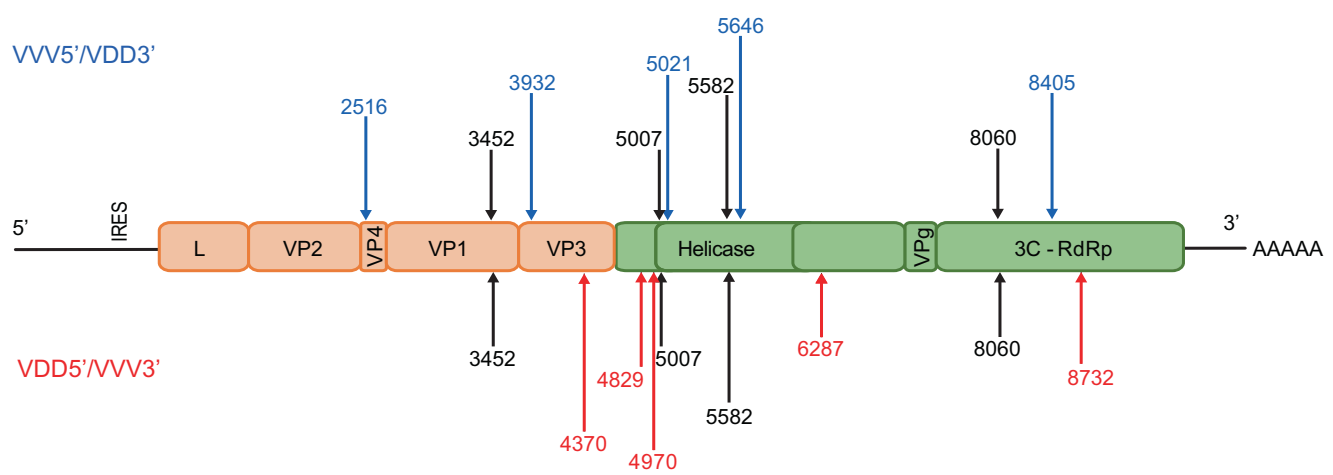

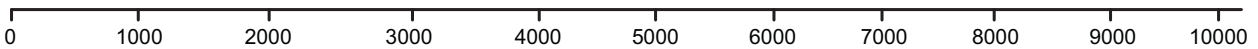

Fig. 5 Genomic recombination events observed between VVV and VDD DWV variants in a superinfected honey bee pupa. a Mapped recombination events in a honey bee pupa initially infected with VVV and superinfected with VDD DWV. The plot shows recombination events occurring along the full length of the DWV genome, with the VDD genome length shown on the $Y$-axis and VVV shown on the $X$-axis. Each bubble represents a unique recombination site and bubble size is determined by the number of mapped and aligned reads for this site obtained using ViReMa analysis. The colour of the bubble indicates the direction of recombination, with blue representing VVV as $5^{\prime}$-acceptor and VDD as $3^{\prime}$-donor and those in red with VDD as $5^{\prime}$-acceptor and VVV as $3^{\prime}$-donor. $\mathbf{b}$ The most frequently observed recombination junctions in all pupal samples analysed, shown at the recombination junction between VVV and VDD genomes. The junctions shown in black occurred with similar frequency in both directions. Those shown in blue occurred predominantly with VVV as $5^{\prime}$-acceptor and VDD as $3^{\prime}$-donor and those in red with VDD as 5'-acceptor and VVV as 3'-donor sequences.

virus. There are at least two distinct types of DWV circulating globally-type A and type B-with documented differences in their distribution [8, 18, 22] and, perhaps, pathogenesis $[13,19,23,25]$. If the outcome of superinfection always favoured one virus type it would influence transmission of DWV variants potentially accounting for their geographic distribution and-if associated with differences in virulence-the impact on the honey bees.

SIE has been reported for DWV, with the suggestion that bees bearing a type $B$ virus were protected from subsequent type $A$ transmitted from infesting Varroa mites [10]. SIE is described for several human, animal and plant viruses [26-35], and may operate via a number of molecular mechanisms [26, 27, 30, 31, 47-50]. Precedents already exist in plants with milder forms of a virus providing protection against more virulent strains $[51,52]$ and the recent spread of DWV type B in the USA $[8,22]$ could be interpreted as an indirect consequence of $\mathrm{SIE}$, with bees harbouring this virus less susceptible to infection by DWV type A. However, there are other potential differences between DWV types such as the ability of variants with type B capsid to replicate in Varroa [25,53], which may enhance its spread over the nonpropagative transmission reported for type A [54].

The availability of a RG system allowed us to investigate the consequences of coinfection and superinfection with DWV type A 
and $B$ in individual honey bees. We found that when coinfected DWV type $A$ and $B$ (VDD and VVV variants) demonstrate broadly similar levels of replication (Fig. 1b). In contrast, in sequential infections, either of virus-fed larvae or injected pupae, superinfecting DWV variants showed delayed replication (Fig. 1). This delay was dependent upon the genetic similarity of the primary and secondary viruses and appeared transient in certain pairings. In genetically divergent pairings (e.g. "VDD $\rightarrow$ VV" and "VVV $\rightarrow$ VDD") high levels of both viruses were reached after a prolonged incubation period. In contrast, where the extent of genetic identity between the primary and secondary virus was greater, the superinfecting virus failed to 'catch up', even after 7 days (Fig. 2). This was most dramatically demonstrated using two genomes that differed by just four nucleotides $\left(\mathrm{VVD}_{\mathrm{S}}\right.$ and $\mathrm{VVD}_{\mathrm{H}}$ variants), in which case the superinfecting virus remained undetectable after 6 days incubation (Fig. S5). In addition, we found that delayed accumulation of the genetically similar superinfecting DWV variants is not specific to honey bee host and was also observed in bumble bees, a species susceptible to DWV infection when directly injected (Fig. S4) [38].

We extended our analysis in honey bee pupae using reporter gene-expressing viruses and demonstrated that replication, characterised by the expression of the fluorescent protein, was inversely related to the level of genetic identity between the primary and superinfecting viruses (Fig. 3). For example, $\mathrm{VDD}_{\mathrm{E}}$ replicated extensively, albeit somewhat delayed when compared with $\mathrm{VDD}_{\mathrm{E}}$-only infected pupae, in pupae that had received VV as the primary virus (Fig. $3 \mathrm{j}-\mathrm{l}$ ), but was undetectable in pupae initially inoculated with VDD (Fig. 3p-s). Notably, in each case where the superinfecting virus showed reduced replication after extended incubation, dominance in the replication showed no directionality according to virus type and was due solely to the order of addition. Based on this data it is likely that sequential infection with DWV type A and B will result in both viruses replicating to maximal levels before eclosion of either worker or drone brood pupae (which pupate for $\sim 12$ or $\sim 14$ days respectively). It remains to be determined whether the delay we demonstrate is sufficient to influence the colony-level virus population, or that carried and transmitted by Varroa.

Where cellular coinfection occurs, viruses have the opportunity to genetically recombine. This is a widespread phenomenon in the single-stranded positive-sense RNA viruses $[55,56]$ and has previously been documented in DWV [6-9]. Our microscopy analysis of honey bee pupae infected with two reporterexpressing DWV variants predominantly demonstrated noncolocalised expression of the fluorescent signal. However, small numbers of dual-infection foci were detected, directly implying that the opportunity for recombination exists (Fig. 4c and Fig. S8). Using next generation sequencing we confirmed the formation of recombinants and characterised the recombination products by analysis of the viral RNA in pupae reciprocally superinfected with VDD and VV. $1-2.2 \%$ of mapped reads spanned recombination junctions, with no evidence for any bias in their directionality (VDD/VVV or VVV/VDD; Fig. S9). Although these junctions mapped throughout the DWV genome, the greatest number were concentrated in the region of the genome encoding the junction of the structural and non-structural proteins (Fig. 5a). This observation matches that found for other picornaviruses and reflects the mix'n'match modular nature of the Picornavirales genome $[6,7,9,18,21]$. In this, functional capsid-coding modules can, through recombination, be juxtaposed with non-structural coding modules from a different parental genome [57]. A small number of recombination junctions ( 350 of 35750 unique junctions mapped) plotted as outliers from the diagonal of genome-length recombinants. Analysis of these sequences showed that the majority were out of frame deletions (Woodford, unpublished), and so incapable of replicating. Our studies using analogous approaches in other RNA viruses show that these types of aberrant products are not unusual and reflect the random nature of the molecular mechanism of recombination [44, 58].

The competition we demonstrate in sequential DWV infections appears to be guided by the amount of genetic identity between the viruses. This suggests it is most likely mediated via RNA interference (RNAi). In arthropods antiviral RNAi response acts via generation of short double stranded RNAs (siRNA) from virus RNA replication intermediates through cleavage by the enzyme Dicer. These are further used by the RNA induced silencing complex to target the destruction of complementary sequences [59, 60]. Hence viral RNA genomes exhibiting greater identity are likely to generate higher numbers of cross-reactive siRNAs while the differences between these siRNA and the target virus genome might have a significant impact [18]. Previous analysis of the RNAi population in DWV-infected honey bees demonstrated that $75 \%$ of DWV-specific short RNA are 21/22 mers [21]. Although DWV type $A$ and $B$ exhibit $~ 85 \%$ genetic identity it is not contiguous (Fig. S1), but is instead distributed in $~ 1350$ short regions of 1-389 nucleotides. Less than $4 \%$ of these identical regions are 21 nucleotides or longer, and therefore capable of generating perfectly complementary siRNAs. Recalculation of the identity between genomes having excluded sequences under 21 nucleotides demonstrates that there is only $34 \%$ genetic identity between DDD and VV (Table S4). Comparing the figures from this analysis and the quantification of DWV accumulation in superinfected pupae suggests a clear relationship between the extent of the competition observed and the genetic identity of contiguous sequences. It is already known that exogenous RNAi can control DWV and other RNA viruses of honey bees [61-63], and in our preliminary studies we have shown that RNAi-mediated suppression of Dicer leads to both increased pathogenesis and viral loads in DWV-infected bees (Fig. S10). Further research will be required to determine the impact of RNAi in competition between superinfecting DWV variants and its potential exploitation in studies to develop cross-reactive vaccines against DWV [36]. These future studies will need to take account of the disrupted complementarity between the genomes (Table S4), the uneven distribution of the RNAi response mapped to the genomic RNA of the infecting virus [21], and both the variation acceptable within the RNAi seed sequence and the RNA structure of the target. Sequential or simultaneous infections with DWV is an inevitable consequence of the multiple routes by which the virus can be acquired-vertically, horizontally per os and vectored by Varroa. Our studies demonstrate that it is the order of acquisition, not the specific type of DWV, that determines the outcome of sequential or superinfection. The role of genetic identity in competition between DWV variants at an individual and landscape scale is likely to be a fruitful area of research, and may allow the future development of rationally designed vaccines against DWV that exploit the conserved RNAi response of the infected host.

\section{REFERENCES}

1. Honey: market value worldwide 2007-2016. https://www.statista.com/statistics/ 933928/global-market-value-of-honey/. Accessed Nov 2020.

2. Highfield AC, El Nagar A, Mackinder LCM, Noël LM-LJ, Hall MJ, Martin SJ, et al. Deformed wing virus implicated in overwintering honeybee colony losses. Appl Environ Microbiol. 2009;75:7212-20.

3. Ongus JR, Peters D, Bonmatin JM, Bengsch E, Vlak JM, van Oers MM. Complete sequence of a picorna-like virus of the genus Iflavirus replicating in the mite Varroa destructor. J Gen Virol. 2004;85:3747-55.

4. Lanzi G, Miranda JRD, Boniotti MB, Cameron CE, Lavazza A, Capucci L, et al. Molecular and biological characterization of Deformed wing virus of honeybees (Apis mellifera L.). J Virol. 2006;80:4998-5009.

5. Fujiyuki T, Takeuchi $H$, Ono M, Ohka S, Sasaki T, Nomoto A, et al. Kakugo virus from brains of aggressive worker honeybees. Adv Virus Res. 2005;65:1-27.

6. Dalmon A, Desbiez C, Coulon M, Thomasson M, Le Conte Y, Alaux C, et al. Evidence for positive selection and recombination hotspots in Deformed wing virus (DWV). Sci Rep. 2017;7:41045 
7. Zioni N, Soroker V, Chejanovsky N. Replication of Varroa destructor virus 1 (VDV1) and a Varroa destructor virus 1-deformed wing virus recombinant (VDV1-DWV) in the head of the honey bee. Virology. 2011;417:106-12.

8. Ryabov EV, Childers AK, Chen Y, Madella S, Nessa A, Vanengelsdorp D, et al. Recent spread of Varroa destructor virus - 1, a honey bee pathogen, in the United States. Sci Rep. 2017;7:17447.

9. Moore J, Jironkin A, Chandler D, Burroughs N, Evans DJ, Ryabov EV. Recombinants between Deformed wing virus and Varroa destructor virus-1 may prevail in Varroa destructor-infested honeybee colonies. J Gen Virol. 2011;92:156-61.

10. Mordecai GJ, Brettell LE, Martin SJ, Dixon D, Jones IM, Schroeder DC. Superinfection exclusion and the long-term survival of honey bees in Varroa-infested colonies. ISME J. 2015;10:1182-91.

11. Woodford L, Evans DJ. Deformed wing virus: using reverse genetics to tackle unanswered questions about the most important viral pathogen of honey bees. FEMS Microbiol Rev. 2020; fuaa070, https://doi.org/10.1093/femsre/fuaa070.

12. Mordecai GJ, Wilfert L, Martin SJ, Jones IM, Schroeder DC. Diversity in a honey bee pathogen: first report of a third master variant of the Deformed Wing Virus quasispecies. ISME J. 2016;10:1264-73.

13. McMahon DP, Natsopoulou ME, Doublet V, Fürst M, Weging S, Brown MJF, et al. Elevated virulence of an emerging viral genotype as a driver of honeybee loss. Proc Biol Sci. 2016;283:443-9.

14. Wilfert L, Long G, Leggett HC, Schmid-Hempel P, Butlin R, Martin SJM, et al. Deformed wing virus is a recent global epidemic in honeybees driven by Varroa mites. Science. 2016;351:594-7.

15. de Miranda JR, Genersch E. Deformed wing virus. J Invertebr Pathol. 2010;103: S48-S61.

16. Roberts JMK, Anderson DL, Durr PA. Absence of deformed wing virus and Varroa destructor in Australia provides unique perspectives on honeybee viral landscapes and colony losses. Sci Rep. 2017;7:6925.

17. Yue C, Schröder M, Gisder S, Genersch E. Vertical-transmission routes for deformed wing virus of honeybees (Apis mellifera). J Gen Virol. 2007;88:2329-36.

18. Ryabov EV, Childers AK, Lopez D, Grubbs K, Posada-Florez F, Weaver D, et al. Dynamic evolution in the key honey bee pathogen deformed wing virus: novel insights into virulence and competition using reverse genetics. PLoS Biol. 2019; 17; https://doi.org/10.1371/journal.pbio.3000502.

19. Martin SJ, Highfield AC, Brettell L, Villalobos EM, Budge GE, Powell M, et al. Global honey bee viral landscape altered by a parasitic mite. Science. 2012;336:1304-6.

20. Loope KJ, Baty JW, Lester PJ, Wilson Rankin EE. Pathogen shifts in a honeybee predator following the arrival of the Varroa mite. Proc Biol Sci. 2019;286:20182499.

21. Ryabov EV, Wood GR, Fannon JM, Moore JD, Bull JC, Chandler D, et al. A virulent strain of deformed wing virus (DWV) of honeybees (Apis mellifera) prevails after Varroa destructor-mediated, or in vitro, transmission. PLoS Pathog. 2014;10: e1004230.

22. Kevill JL, de Souza FS, Sharples C, Oliver R, Schroeder DC, Martin SJ. DWV-A lethal to honey bees (Apis mellifera): a colony level survey of DWV variants (A, B, and C) in England, Wales, and 32 States across the US. Viruses. 2019;11:426.

23. Tehel $A, V u$ Q, Bigot $D$, Gogol-Döring $A$, Koch $P$, Jenkins $C$, et al. The two prevalent genotypes of an emerging infectious disease, Deformed wing virus, cause equally low pupal mortality and equally high wing deformities in host honey bees. Viruses. 2019;11:114.

24. Norton AM, Remnant EJ, Buchmann G, Beekman M. Accumulation and competition amongst Deformed wing virus genotypes in naïve Australian honeybees provides insight Into the increasing global prevalence of genotype B. Front Microbiol. 2020;11:620.

25. Gusachenko ON, Woodford L, Balbirnie-Cumming K, Campbell EM, Christie CR, Bowman AS, et al. Green bees: reverse genetic analysis of Deformed wing virus transmission, replication, and tropism. Viruses. 2020;12:532.

26. Steck FT, Rubin $\mathrm{H}$. The mechanism of interference between an avian leukosis virus and Rous sarcoma virus. II. Early steps of infection by RSV of cells under conditions of interference. Virology. 1966;29:642-53.

27. Adams RH, Brown DT. BHK cells expressing Sindbis virus-induced homologous interference allow the translation of nonstructural genes of superinfecting virus. J Virol. 1985;54:351-7.

28. Strauss JH, Strauss EG. The alphaviruses: gene expression, replication, and evolution. Microbiol Rev. 1994;58:491-562.

29. Karpf AR, Lenches E, Strauss EG, Strauss JH, Brown DT. Superinfection exclusion of alphaviruses in three mosquito cell lines persistently infected with Sindbis virus. J Virol. 1997;71:7119-23.

30. Singh IR, Suomalainen M, Varadarajan S, Garoff $H$, Helenius A. Multiple mechanisms for the inhibition of entry and uncoating of superinfecting Semliki Forest virus. Virology. 1997;231:59-71.

31. Geib T, Sauder C, Venturelli S, Hässler C, Staeheli P, Schwemmle M. Selective virus resistance conferred by expression of Borna disease virus nucleocapsid components. J Virol. 2003;77:4283-90.
32. Edwards MC, Bragg J, Jackson AO. Natural resistance mechanisms to viruses in barley. In: Loebenstein G and Carr JP, editors. Natural Resistance Mechanisms of Plants to Viruses. Dordrecht, The Netherlands: Springer; 2006. p. 465-501.

33. Bergua M, Zwart MP, El-Mohtar C, Shilts T, Elena SF, Folimonova SY. A viral protein mediates superinfection exclusion at the whole-organism level but is not required for exclusion at the cellular Level. J Virol. 2014;88:11327-38.

34. Michel N, Allespach I, Venzke S, Fackler OT, Keppler OT. The Nef protein of human immunodeficiency virus establishes superinfection immunity by a dual strategy to downregulate cell-surface CCR5 and CD4. Curr Biol. 2005;15:714-23.

35. Tscherne DM, Evans MJ, von Hahn T, Jones CT, Stamataki Z, McKeating JA, et al. Superinfection exclusion in cells infected with hepatitis C virus. J Virol. 2007;81:3693-703.

36. Leonard SP, Powell JE, Perutka J, Geng P, Heckmann LC, Horak RD, et al. Engineered symbionts activate honey bee immunity and limit pathogens. Science. 2020;367:573-6.

37. Lamp B, Url A, Seitz K, Rgen Eichhorn J, Riedel C, Sinn LJ, et al. Construction and rescue of a molecular clone of Deformed wing virus (DWV). PLOS ONE. 2016;11: e0164639.

38. Gusachenko ON, Woodford L, Balbirnie-Cumming K, Ryabov EV, Evans DJ. Evidence for and against deformed wing virus spillover from honey bees to bumble bees: a reverse genetic analysis. Sci Rep. 2020;10:16847.

39. Routh A, Johnson JE. Discovery of functional genomic motifs in viruses with ViReMa - a Virus Recombination Mapper - for analysis of next-generation sequencing data. Nucleic Acids Res. 2014;42:e11.

40. Ryabov EV, Christmon K, Heerman MC, Posada-Florez F, Harrison RL, Chen Y, et al. Development of a honey bee RNA virus vector based on the genome of a Deformed wing virus. Viruses. 2020;12:374.

41. Mueller S, Wimmer E. Expression of foreign proteins by poliovirus polyprotein fusion: analysis of genetic stability reveals rapid deletions and formation of cardioviruslike open reading frames. J Virol. 1998;72:20-31.

42. Kirkegaard K, Baltimore D. The mechanism of RNA recombination in poliovirus. Cell. 1986;47:433-43.

43. Egger D, Bienz K. Recombination of poliovirus RNA proceeds in mixed replication complexes originating from distinct replication start sites. J Virol. 2002;76:10960-71.

44. Lowry K, Woodman A, Cook J, Evans DJ. Recombination in enteroviruses is a biphasic replicative process involving the generation of greater-than genome length 'imprecise' Intermediates. PLoS Pathog. 2014;10; https://doi.org/10.1371/ journal.ppat.1004191.

45. de Miranda JR, Fries I. Venereal and vertical transmission of deformed wing virus in honeybees (Apis mellifera L.). J Invertebr Pathol. 2008;98:184-9.

46. Yañez O, Jaffé R, Jarosch A, Fries I, Robin FAM, Robert JP, et al. Deformed wing virus and drone mating flights in the honey bee (Apis mellifera): Implications for sexual transmission of a major honey bee virus. Apidologie. 2012;43:17-30.

47. Simon KO, Cardamone JJ Jr, Whitaker-Dowling PA, Youngner JS, Widnell CC. Cellular mechanisms in the superinfection exclusion of vesicular stomatitis virus. Virology. 1990;177:375-9.

48. Stevenson M, Meier C, Mann AM, Chapman N, Wasiak A. Envelope glycoprotein of HIV induces interference and cytolysis resistance in CD4+ cells: mechanism for persistence in AIDS. Cell. 1988;53:483-96.

49. Bratt MA, Rubin H.Specific interference among strains of Newcastle disease virus. II. Comparison of interference by active and inactive virus.Virology. 1968:35:381-94.

50. Zou G, Zhang B, Lim P-Y, Yuan Z, Bernard KA, Shi P-Y. Exclusion of West Nile virus superinfection through RNA replication. J Virol. 2009;83:11765-76.

51. Ziebell H, Carr JP. Cross-protection: a century of mystery. Adv Virus Res. 2010;76:211-64.

52. Folimonova SY. Developing an understanding of cross-protection by Citrus tristeza virus. Front Microbiol. 2013;4; https://doi.org/10.3389/fmicb.2013.00076.

53. Gisder $S$, Genersch E. Direct evidence for infection of mites with the beepathogenic Deformed wing virus variant B - but not variant A - via fluorescencehybridization analysis. J Virol. 2021;95:e01786-20.

54. Posada-Florez F, Childers AK, Heerman MC, Egekwu NI, Cook SC, Chen Y, et al. Deformed wing virus type $A$, a major honey bee pathogen, is vectored by the mite Varroa destructor in a non-propagative manner. Sci Rep. 2019;9:12445.

55. Barr JN, Fearns R. How RNA viruses maintain their genome integrity. J Gen Virol. 2010;91:1373-87.

56. Bentley K, Evans DJ. Mechanisms and consequences of positive-strand RNA virus recombination. J Gen Virol. 2018;99:1345-56.

57. Muslin C, Mac Kain A, Bessaud M, Blondel B, Delpeyroux F. Recombination in enteroviruses, a multi-step modular evolutionary process. Viruses. 2019;11:859.

58. Alnaji FG, Bentley K, Pearson A, Woodman A, Moore JD, Fox H, et al. Recombination in enteroviruses is a ubiquitous event independent of sequence homology and RNA structure. 2020; preprint at bioRxiv; https://doi.org/10.1101/ 2020.09.29.319285 
59. Brutscher LM, Flenniken ML. RNAi and antiviral defense in the honey bee. J Immunol Res. 2015;2015:941897.

60. Chejanovsky N, Ophir R, Schwager MS, Slabezki Y, Grossman S, Cox-Foster D. Characterization of viral siRNA populations in honey bee colony collapse disorder. Virology. 2014;454-5:176-83.

61. Desai SD, Eu YJ, Whyard S, Currie RW. Reduction in deformed wing virus infection in larval and adult honey bees (Apis mellifera L.) by double-stranded RNA ingestion. Insect Mol Biol. 2012;21:446-55.

62. Hunter W, Ellis J, Vanengelsdorp D, Hayes J, Westervelt D, Glick E, et al. Largescale field application of RNAi technology reducing Israeli acute paralysis virus disease in honey bees (Apis mellifera, hymenoptera: Apidae). PLoS Pathog. 2010;6:e1001160.

63. Maori E, Paldi N, Shafir S, Kalev H, Tsur E, Glick E, et al. IAPV, a bee-affecting virus associated with colony collapse disorder can be silenced by dsRNA ingestion. Insect Mol Biol. 2009;18:55-60.

\section{ACKNOWLEDGEMENTS}

We express our gratitude to Dr Marcus Bischoff and Gill McVee (University of St Andrews) for helping with microscopic imaging, Ashley Pearson (University of St Andrews) for assistance in molecular biology assays. This work was supported by grant funding from BBSRC BB/M00337X/2 and BB/I000828/1.

\section{DECLARATIONS (ETHICS):}

\section{COMPETING INTERESTS}

The authors declare no competing interests.

\section{ADDITIONAL INFORMATION}

Supplementary information The online version contains supplementary material available at https://doi.org/10.1038/s41396-021-01043-4.

Correspondence and requests for materials should be addressed to O.N.G.

Reprints and permission information is available at http://www.nature.com/ reprints

Publisher's note Springer Nature remains neutral with regard to jurisdictional claims in published maps and institutional affiliations.

(i) Open Access This article is licensed under a Creative Commons Attribution 4.0 International License, which permits use, sharing, adaptation, distribution and reproduction in any medium or format, as long as you give appropriate credit to the original author(s) and the source, provide a link to the Creative Commons license, and indicate if changes were made. The images or other third party material in this article are included in the article's Creative Commons license, unless indicated otherwise in a credit line to the material. If material is not included in the article's Creative Commons license and your intended use is not permitted by statutory regulation or exceeds the permitted use, you will need to obtain permission directly from the copyright holder. To view a copy of this license, visit http://creativecommons. org/licenses/by/4.0/.

(c) The Author(s) 2021 